SECTION V

THE SOLAR FLARE PLASMA

Chairman:

M. KUPERUS 


\title{
LABORATORY-PRODUCED RADIATION RELATED TO THE SOLAR FLARE EMISSION
}

\author{
R. C. ELTON and T. N. LIE \\ U.S. Naval Research Laboratory, Washington, D.C. 20390, U.S.A.
}

\begin{abstract}
A review is given of continuing efforts to generate laboratory spectra at photon energies above $1 \mathrm{keV}$ from highly ionized atoms. Emphasis is placed on the most easily-interpreted highest ion stages such as the hydrogenic, helium-like and lithium-like species. The close similarities to solar flare observations of certain signatures, such as the line and continuum X-ray spectra and microwave emission as well as their sequence of occurrence, are pointed out and scaling factors are given.
\end{abstract}

\section{Introduction}

There clearly exists an increasing need for laboratory data to support the interpretation of the less-accessible astrophysical data. Much of the recent interesting solar data have been from the dynamic flare regions with transient non-thermal mechanisms present which are often associated with violent plasma instabilities. With the high temperatures present, much of the data unique to these flare regions are most readily signified at X-ray to $\gamma$-ray energies. However, there is also the strong radio emission in the microwave region closely associated with the $\mathrm{X}$-ray impulses. To understand the origins of the observed phenomena clearly involves an improved basic physics understanding such as can be provided in a laboratory-scale experiment. It is the purpose of the present review to point out how certain laboratory experiments have kept pace with the astrophysical progress and the close parallels that exist, in spite of the large scaling necessary for the reduced size and increased particle density in the laboratory.

The review which follows will concern two particular devices which are at present the most useful for the X-ray photon energy range above $1 \mathrm{keV}$, namely the condensed vacuum spark and the coaxial gun gaseous discharge (plasma focus).* The emphasis will be on the first device for which the authors are most experienced.

\section{Vacuum Spark}

\section{A. EARLY EFFORTS}

The vacuum spark has been an extremely valuable tool for producing laboratory spectra from highly ionized atoms in the vacuum ultraviolet and X-ray regions since the pioneering efforts of Edlén (1947) and colleagues in the 1930's. Flemberg (1942) managed

* Plasmas with somewhat similar characteristics are generated by focusing a high power laser beam onto a target. Line spectra of atoms ionized up to seventeen times have been produced with photons so far not exceeding $1 \mathrm{keV}$ in energy. There are, however, some preliminary reports of additional radiation with energies in the hundreds-of-keV range from such devices, where very short laser pulses are used. 
to extend these studies to wavelengths as short as $6.4 \AA$ by using a high voltage $(75 \mathrm{kV})$ capacitive discharge $(0.4 \mu \mathrm{F})$ and by dispersing the radiation with a (concave) curved crystal spectrograph. His spectra included helium-like resonance and associated intercombination lines of $\mathrm{F}, \mathrm{Mg}$, and $\mathrm{Al}$ as well as at least one identified satellite line ( $\mathrm{F}$ ). Noticeably absent from his spectra are lines from the hydrogenic species.

\section{B. PLASMA CONCENTRATION}

\section{Initial Discoveries}

A significant breakthrough in producing higher temperatures and shorter wavelength radiation was achieved by Cohen et al.(1968) who, in the course of taking X-ray pinhole photographs of a somewhat faster vacuum spark discharge, found that the $\mathrm{X}$ radiation predominantly originated at a small region in the electrode gap, namely from a concentrated plasma. This device was powered by a $13 \mu \mathrm{F}$ capacitor charged to $19 \mathrm{kV}$ $(E=2.3 \mathrm{~kJ})$ and delivered a peak current of $176 \mathrm{kA}$. The X-ray emission was of sufficient photon energy to permit open-air diagnostics through a thin (beryllium) window in the vacuum chamber.

The X-ray spectrometry was also made particularly simple by the minute size of the source, so that a flat crystal was used without an entrance slit to disperse the radiation according to the varying incident angle across the surface (see Figure 1). Within the

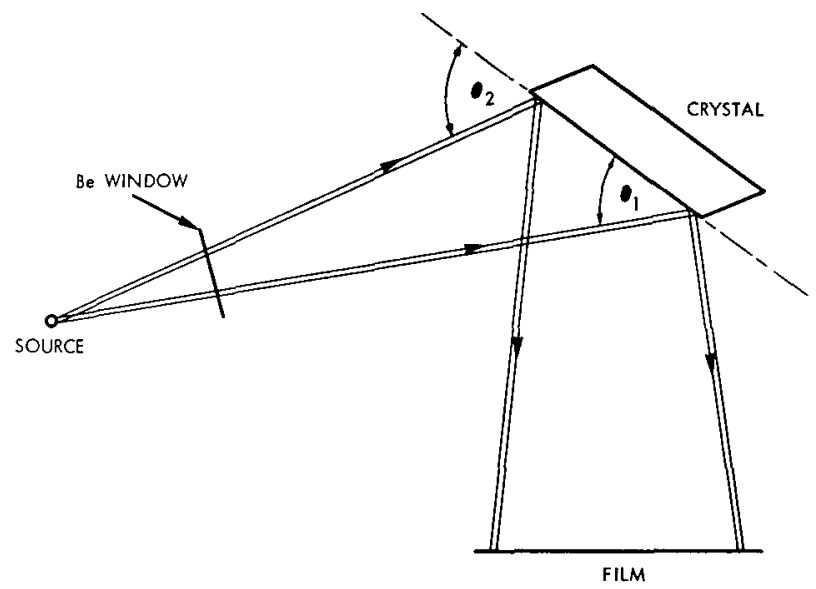

Fig. 1. Schematic of Bragg crystal spectrograph (from Cohen et al., 1968, Figure 2).

somewhat limited resolution achieved, they were able to record the resonance lines of helium-like Ti, V, Fe, Co, and Ni. Their iron spectrum is shown in Figure 2, as is also their tentative identification of the $1 s^{2}-1 s 3 p$ line of helium-like Fexxv. They were also able to record the Lyman- $\alpha$ line of hydrogenic Fexxvi. The results of their wavelength measurements are shown in Figure 3. 


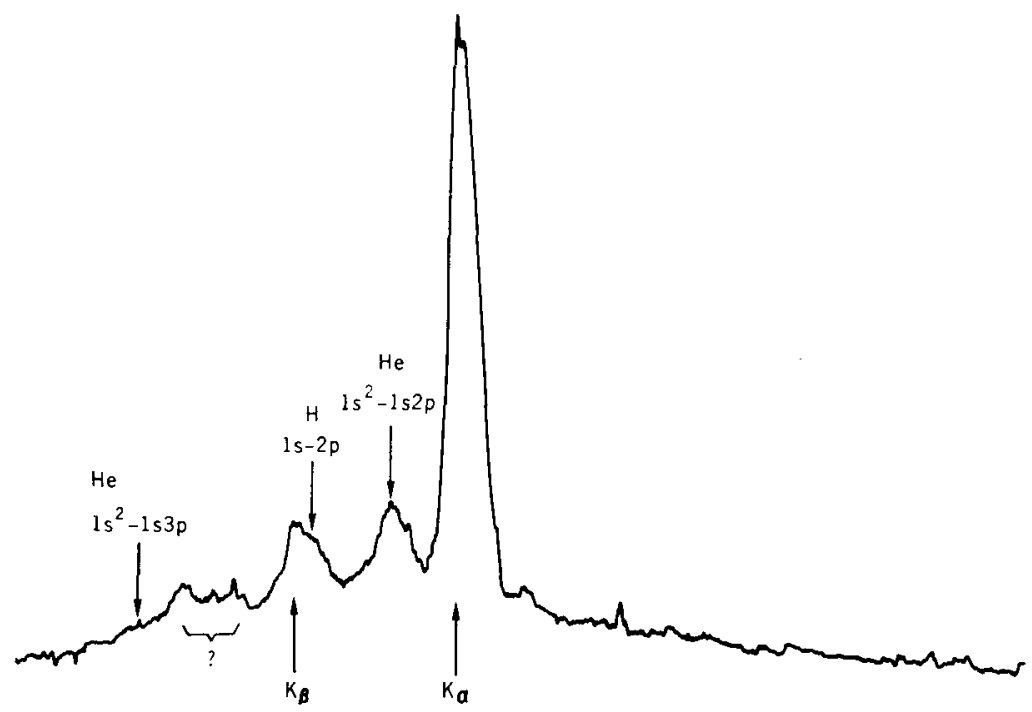

Fig. 2. Microdensitometer tracing of an X-ray spectrogram obtained with 100 discharges using iron electrodes (from Cohen et al., 1968, Figure 5).

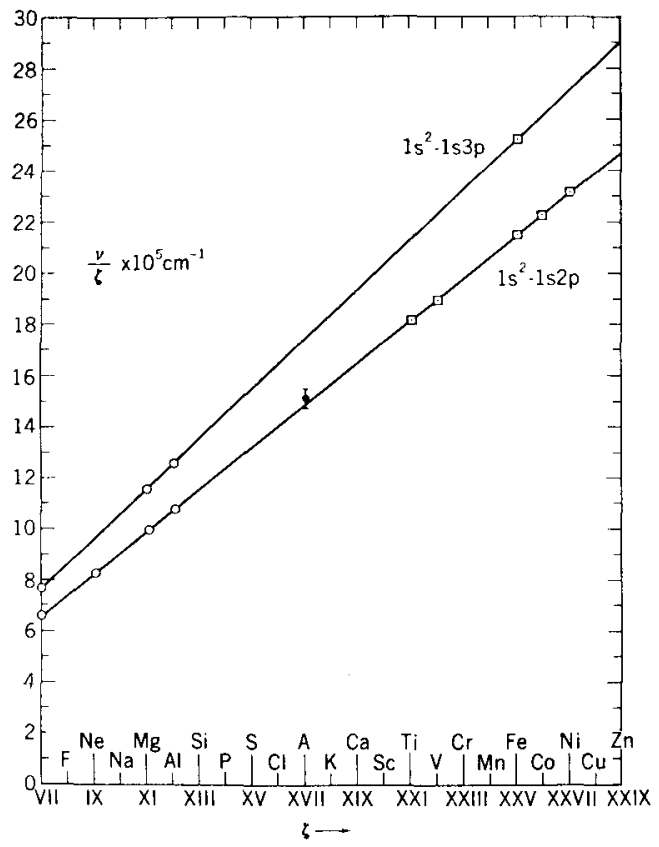

Fig. 3. Helium isoelectronic sequence plot of charge state number $\zeta$ vs. wavenumber divided by $\zeta$. The squares represent data from a vacuum spark (Cohen et al., 1968) while the circles represent previously published data. The Arxvir data arrive from a plasma focus device [see text and Peacock et al. (1969)]. (This figure reproduced from Cohen et al. 1968, Figure 6.) 


\section{Further Efforts}

In an effort to improve the spectral resolution and possibly learn more of the nature of the plasma concentration, this work was extended by Lie and Elton (1971) with a similar device illustrated in Figures 4 and $5\left(C=15 \mu \mathrm{F}, V=14 \mathrm{kV}, E=1.5 \mathrm{~kJ}, I_{\mathrm{p}}=100 \mathrm{kA}\right)$. By incorporating a pointed anode the lateral motion from shot-to-shot was reduced, which resulted in improved spectral resolution for multiple (superimposed) discharges.

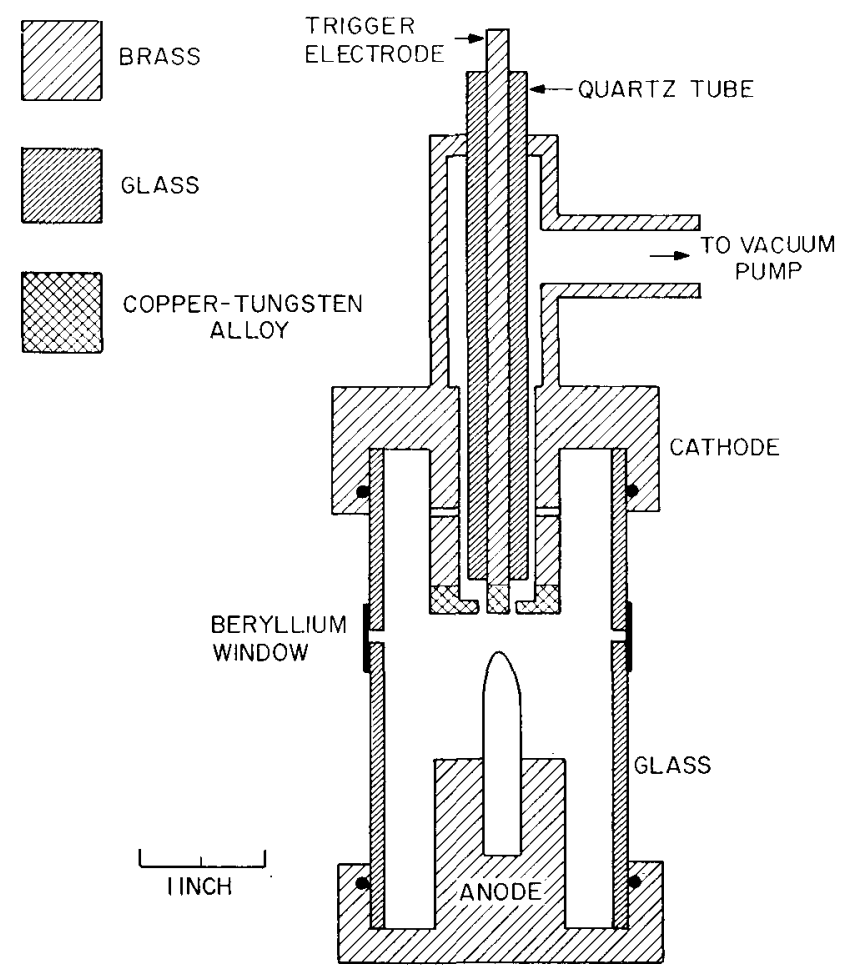

Fig. 4. Schematic diagram of the vacuum spark apparatus (from Lie and Elton, 1971, Figure 1).

A time integrated X-ray pinhole photograph showing the outline of the anode tip as well as the radiation burst (see Figure 13 below) from the concentrated region $(\sim 0.002 \mathrm{~cm})$ is presented in Figure 6 . The concentration of plasma appears from time resolved photographs to occur after the electrode gap initially fills with metallic (anode) plasma, following the traverse of a localized radial pinch from the cathode region to the anode, and just after subsequent radial expansion begins. This latter phase (III) is shown in the streak photographs in Figure 7.

(a) Line Radiation. The resulting time integrated spectrum for 200 discharges with a titanium anode (the anode material was found to dominate the spectrum) is shown in Figure 8 and features, in addition to the helium-like (TixxI) resonance and associated 


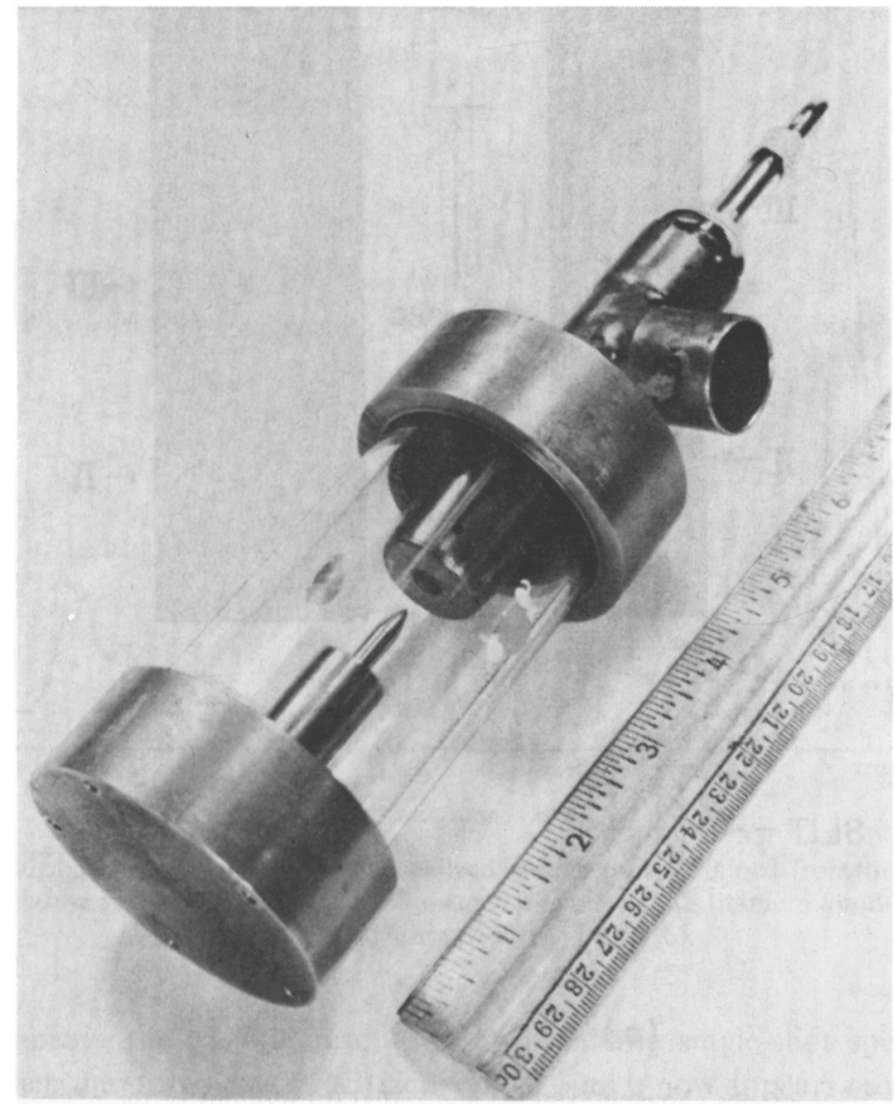

Fig. 5. Photograph of the vacuum spark apparatus.
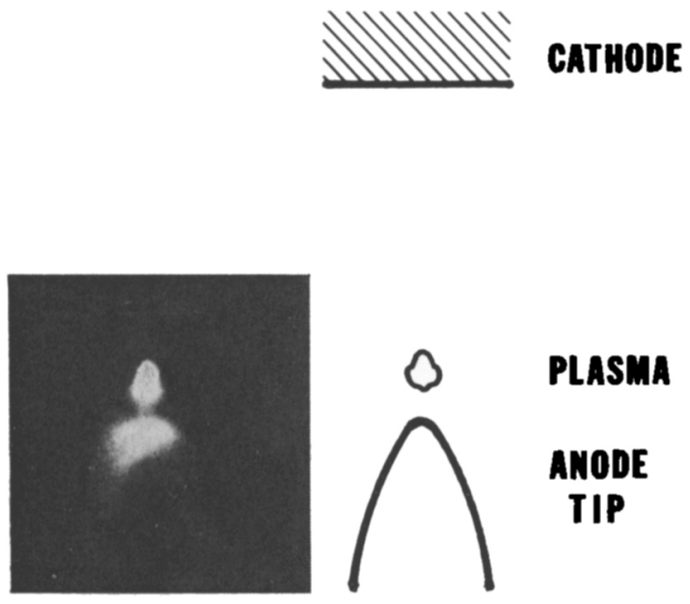

Fig. 6. X-ray pinhole photograph of concentrated plasma near pointed anode tip. 

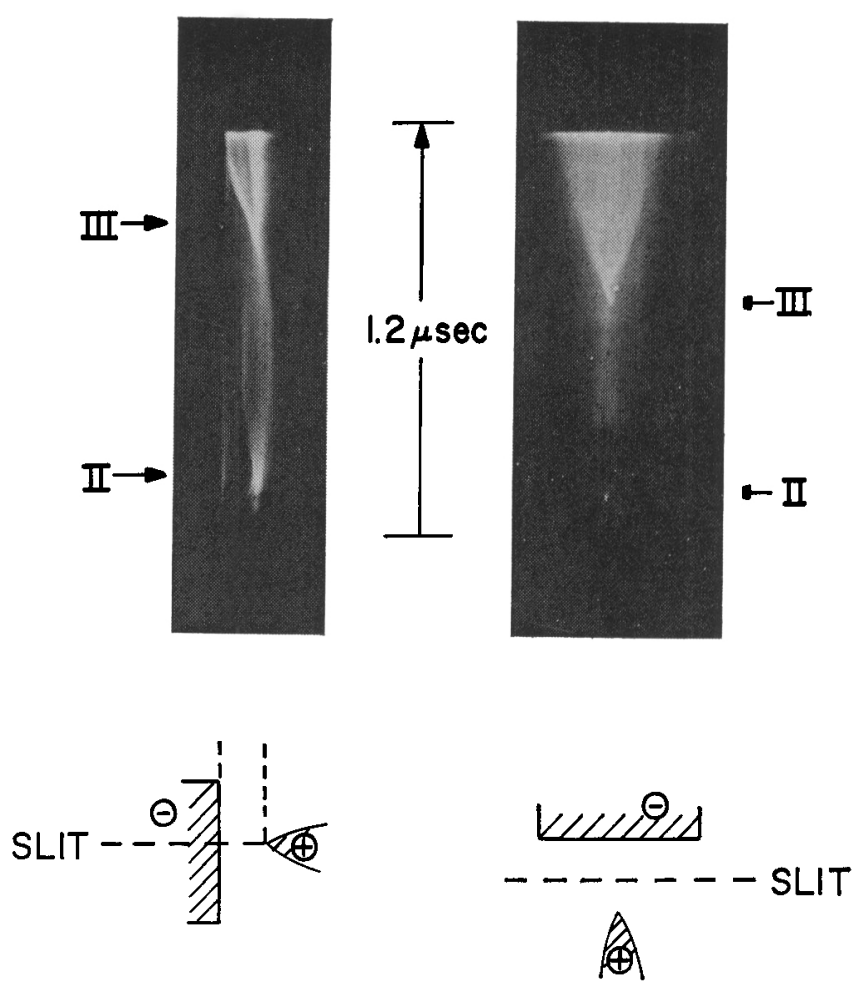

(a)

(b)

Fig. 7. Streak photographs showing axial (a) and radial (b) motions of the plasma column in the vacuum spark device. The alignment of the viewing slit relative to the discharge axis is shown. In (b) the plasma is viewed $2 \mathrm{~mm}$ from the anode tip. The indicated phases of the discharge correspond to those in Figure 13. (Reproduced from Figure 5, Lie and Elton, 1971).

intercombination line, a series of $\mathrm{K} \alpha$-type inner-shell transitions extending from the strong Li-like Tixx ion down to O-like Tixv, below which they blend together. Also shown in Figure 8 is the TixxII hydrogenic Lyman- $\alpha$ doublet, which is considerably weaker than the helium-like resonance lines. This is not yet completely understood without detailed time resolved measurements, i.e., while it could be explained as a marginal confinement time for ionization equilibrium to TixxII, higher stages of ionization in other elements are observed.

Similar results for an iron anode are shown in Figure 9, where the correspondence with solar flare spectra reported by Neupert and Swartz (1970) (NASA) and by Doschek et al. (1971) (NRL) is shown in comparison. The solar flare spectra include the strong $1 s 2 s^{3} S \rightarrow 1 s^{2}{ }^{1} S$ Fexxv transition blended with the Fexxiv lithium-like satellites, which however is not significant at the high electron densities in the laboratory where collisional depopulation dominates.

Finally, with a copper anode, a somewhat weaker spectrum is shown in Figure 10. 


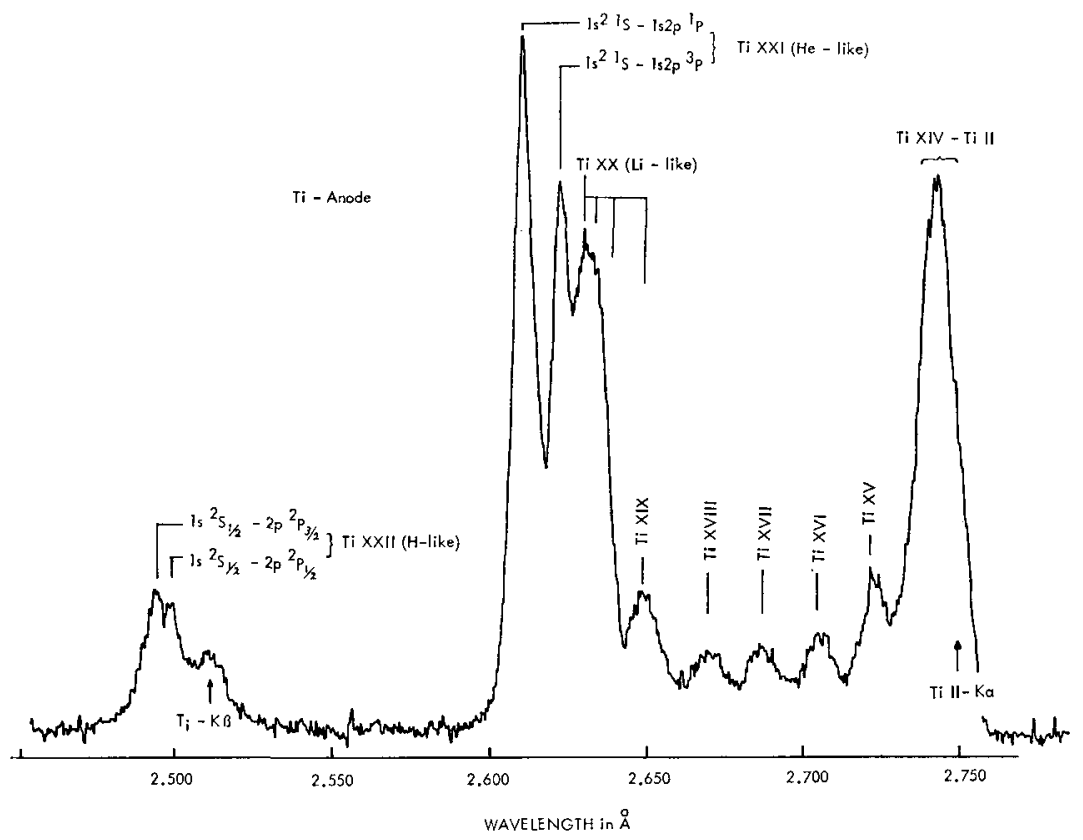

Fig. 8. Microdensitometer scan of a space-resolved spectrogram obtained from the concentrated plasma region (see Figure 11) of a vacuum spark with a titanium anode (from Lie and Elton, 1971,) Figure 2b).

Efforts to improve the spectral resolution by obtaining single-shot spectra are encouraging when the stored energy $(\mathrm{C})$ is increased, and it now appears most likely that the resolution is limited by the (flat) lithium-fluoride crystal used, which was chosen for high reflectivitity at some sacrifice in optimum resolution.*

For wavelength measurement, narrower characteristic $\mathrm{K} \alpha$ and $\mathrm{K} \beta$ lines from the anode tip were used as standards and were isolated from the concentrated plasma blended radiation by the spatial (axial) resolution obtained from a $(1 \mathrm{~mm})$ slit (perpendicular to the discharge axis) placed between the source and the crystal (see Figure 11). This also served to define the plasma source region for the highly ionized species. The wavelength separation from the singlet resonance line of TixxI, Fexxv, and CuxxvIII are compared favorably with theory (House, 1969) in Figure 12.

(b) Continuum Radiation. For wavelengths shorter than the series limit for the hydrogenic species, the continuum has been isolated with metallic filters and observed with and without time resolution. The absolute emission from the concentrated plasma only has yielded an order of magnitude estimate of the electron density of $10^{21} \mathrm{~cm}^{-3}$,

* In a contribution to this conference (paper No. 2.9) Schwob and Fraenkel report spectra of iron and nickel from 2000 discharges in a similar device obtained with a curved crystal spectrograph with a resolution of $3 \times 10^{-4} \AA$ They were thus able to extend the comparison in Figure 12 to the F-like species. 
and relative comparisons through various filters (Elton, 1968) has yielded a shape consistent with an electron kinetic temperature varying most often from $2-10 \mathrm{keV}$ from shot-to-shot. Due mostly to detectors used, these measurements were dominated by the softer component which arrives in three major bursts (I, II, and III) as shown in Figure 13, while the plasma line radiation from highly ionized species is limited to the third burst. Note particularly that the highly ionized species occur very rapidly and this is only barely understandable from simple sequential stepwise ionization, assuming a high initial density; multiple ionization seems quite likely to occur, i.e., via Auger and 'shake-off' type processes.

Finally (as shown also in Figure 13) a much harder X-ray continuum component has been detected through very thick $(3 \mathrm{~mm} \mathrm{~Pb})$ absorbers with an energy as high as $1 \mathrm{MeV}$. The spectral detail remains to be resolved and the burst is considerably shorter than for the softer component. Also, it occurs at the very onset of burst III which is noticed to be quite similar to some recent solar flare observations, where hard X-ray bursts are observed early followed by a slower (softer) component (Kane and Anderson, 1970).

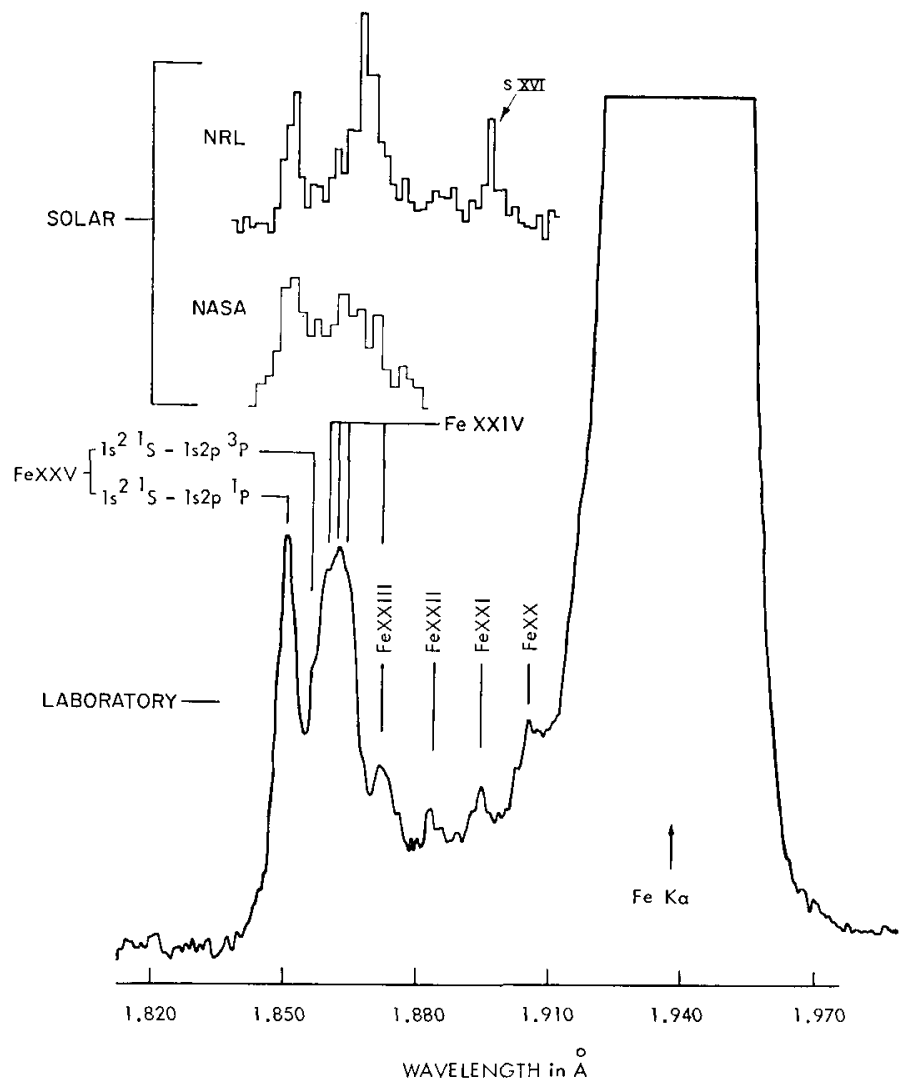

Fig. 9. Microdensitometer scan of a spectrogram obtained with a pointed iron anode in a vacuum spark device (from Lie and Elton, 1971, Figure 2a). Two solar flare spectra, NASA (Neupert and

Schwartz 1970, Figure 2) and NRL (Doschek et al., 1971, Figure 2), are shown for comparison. 
(c) Microwave Radiation. Also noted in the solar flare observations (Kane et al., 1970) is a microwave burst that begins generally with the hard X-ray burst. A search for such radio emission in the laboratory device has indeed revealed (Lie and Elton, 1972) a burst of predominantly $X$ band $(\lambda=3 \mathrm{~cm}, v=10 \mathrm{GHz})$ radiation simultaneous

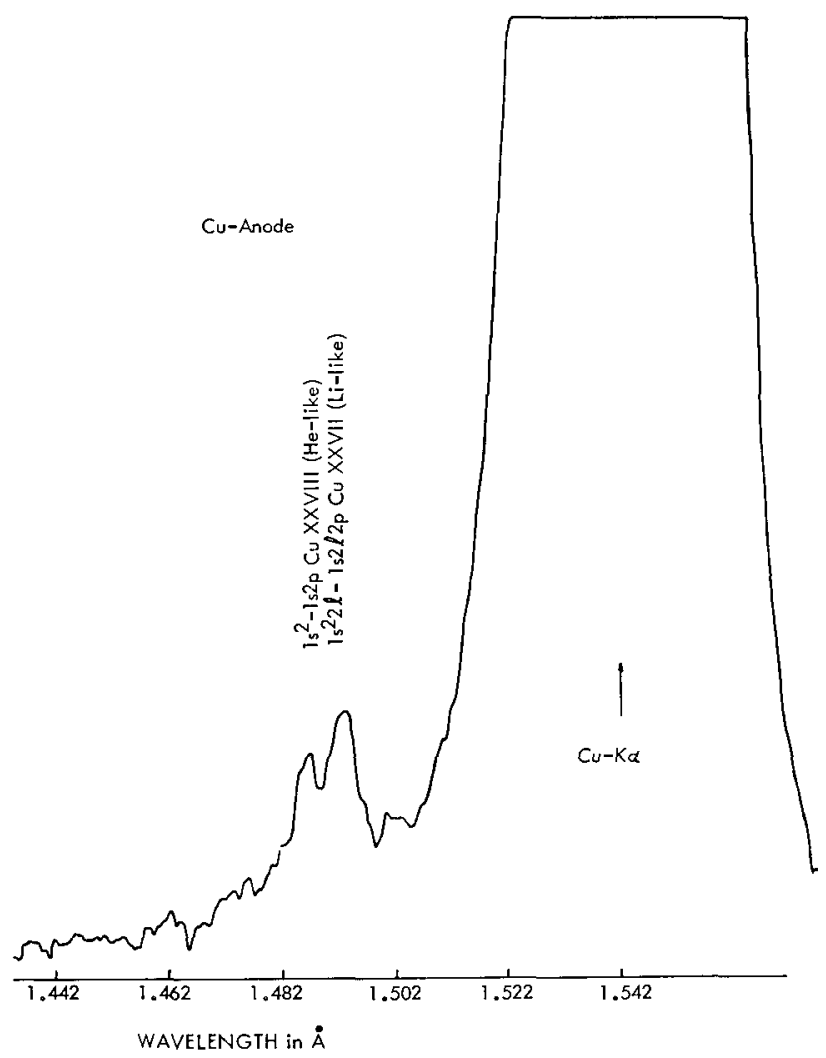

Fig. 10. Microdensitometer scan of a spectrogram obtained with a pointed copper anode in a vacuum spark device.

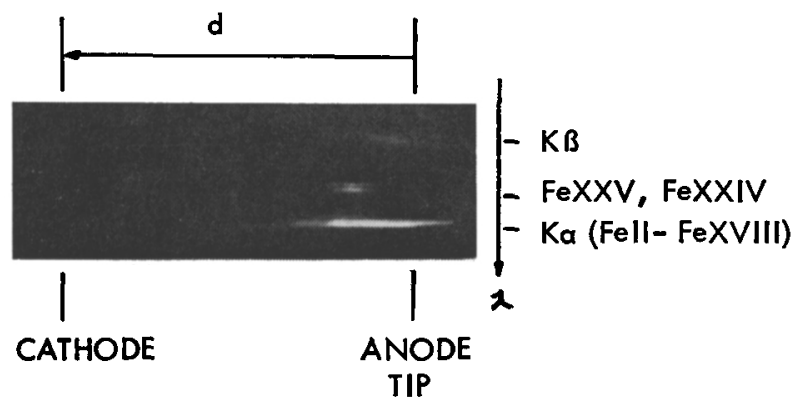

Fig. 11. Space-resolved spectrum obtained through a $1 \mathrm{~mm}$ wide slit aligned perpendicular to the axis of the spark channel. 


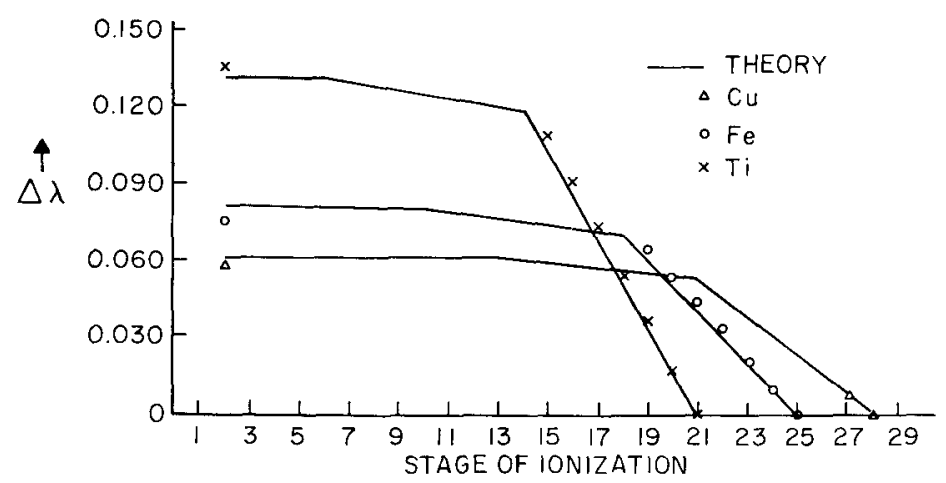

Fig. 12. Comparison between experimental and theoretical (see House, 1969) results for the displacement (in $\AA$ ) of resolved inner-shell lines from the resonance line of the helium-like ions (from Lie and Elton, 1971, Figure 3).

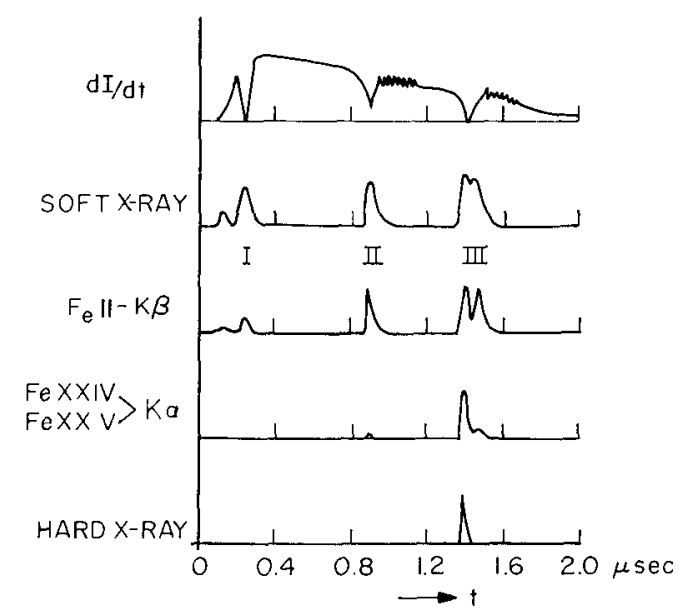

Fig. 13. Oscillograms for the vacuum spark device. The indicated phases (I, II, and III) are as discussed in the text and included in Figure 7 (from Lie and Elton, 1971, Figure 4).

TABLE I

Phenomenological comparison: solar flare and laboratory plasma ${ }^{a}$

\begin{tabular}{llll} 
& Solar flare & $\begin{array}{l}\text { Low-pressure } \\
\text { discharge }\end{array}$ & $\begin{array}{l}\text { scaling } \\
\text { factor }\end{array}$ \\
\hline Electron density & $10^{11} \sim 10^{12} \mathrm{~cm}^{-3}$ & $\sim 10^{21} \mathrm{~cm}^{-3}$ & $10^{-10}-10^{-9}$ \\
Electron temperature & $\sim 1-4 \mathrm{keV}$ & $2-10 \mathrm{keV}$ & $\sim 1$ \\
Soft X-ray risetime & $\sim 2 \mathrm{~min}$ & $1-2 \times 10^{-8} \mathrm{~s}$ & $\sim 10^{10}$ \\
X-ray lifetime & $\sim 3-10 \mathrm{~min}$ & $2-6 \times 10^{-8} \mathrm{~s}$ & $\sim 10^{10}$ \\
Hard X-ray risetime & $\lesssim 10 \mathrm{~s}$ & $\lesssim 10^{-9} \mathrm{~s}^{\mathrm{b}}$ & $\sim 10^{10}$ \\
Velocity of plasma ejection & $1.5 \times 10^{8} \mathrm{~cm} \mathrm{~s}^{-1}$ & $\sim 10^{7} \mathrm{~cm} \mathrm{~s}^{-1}$ & $\sim 10$ \\
Microwaves & up to $90 \mathrm{GHz}$ & $10-36 \mathrm{GHz}$ & $\sim 1$ \\
High energy X-rays & $>500 \mathrm{keV}$ & $>1 \mathrm{MeV}$ & $\sim 1$ \\
\hline
\end{tabular}

a It should be realized that these features are not at all reproducible from flare-to flare and similarly from shot-to-shot in the laboratory case.

b The risetime measurement is limited by the $\mathrm{X}$-ray detector time resolution. 
with the X-ray bursts. That similar radiation is observed from sources of such widely different densities and fields ( $B=10^{7} \mathrm{G}$ in the concentrated spark) is somewhat surprising and indicates a radiation source which is relatively environmentally independent, which for example would not be the case for cyclotron radiation.

The similarities of features in the solar flare and the concentrated spark and scaling factors (largest where size and density are involved) are summarized (Lie and Elton, 1972) in Table I.

\section{Coaxial Gun Plasma Focus}

It is well known by now that a coaxial gun gaseous discharge is capable of producing a dense plasma focus just beyond the tip of the center electrode (anode) as illustrated in the schematic in Figure 14. The characteristics of this device have recently been summarized by Mather (1971). Generally it is a much larger device than the vacuum sparks

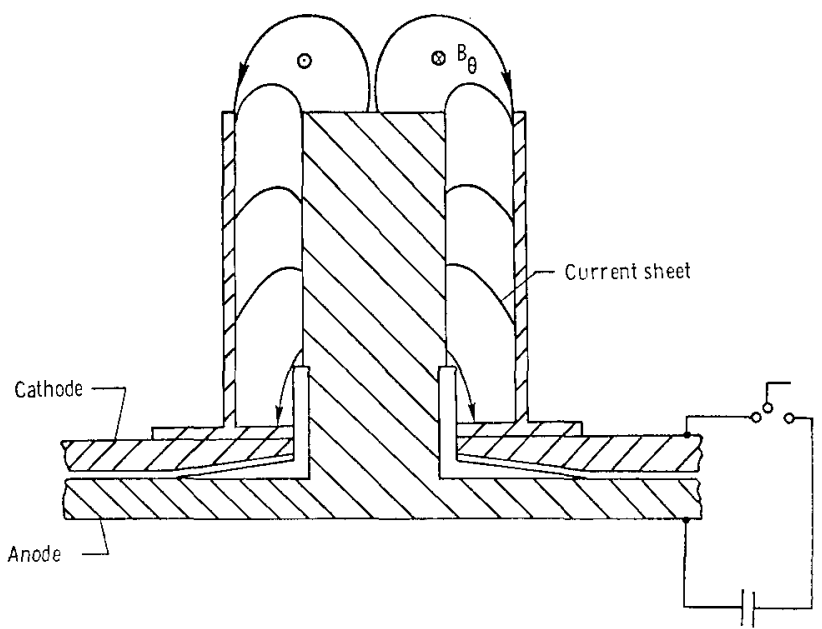

Fig. 14. Simplified schematic diagram of a coaxial gun gaseous discharge (plasma focus) device (from Lee et al., 1971b, Figure 1).

described above (outer diameter typically $10 \mathrm{~cm}$ ), consumes more energy (typically $30-40 \mathrm{~kJ}$ ) and operates as a gas discharge in contrast to the vacuum spark which produces mostly electrode (anode) spectra.* The total X-ray flux is probably $1-2$ orders of the magnitude greater than from present vacuum sparks. Also, the plasma no longer represents a point source and spectroscopy with high resolution requires a narrow entrance slit, so that typically ten discharges are required per exposure.

* Survey studies in the grazing incidence region with the vacuum spark described above indicate considerable oxygen and carbon lines, probably arising from gases released from the main electrodes and the trigger system materials during the initial breakdown. The extent of these elements, particularly in the concentrated region, is not known at present. 
(a) Line Radiation. Peacock and colleagues have studied extensively the spectra of highly stripped neon and argon which are added in small amounts to the deuterium ambient gas. Using grazing incidence spectroscopy at very small (10 min) angles of incidence, Peacock et al. (1969) have succeeded in observing, in addition to Ne IX and $\mathrm{X}$ resonance lines, ArXVII and ArXVIII lines at $3.9 \AA$ with several superimposed discharges. Using a concave curved mica crystal, Peacock et al. (1971) have very recently reported (see Figure 15) well-resolved resonance and intercombination lines of helium-

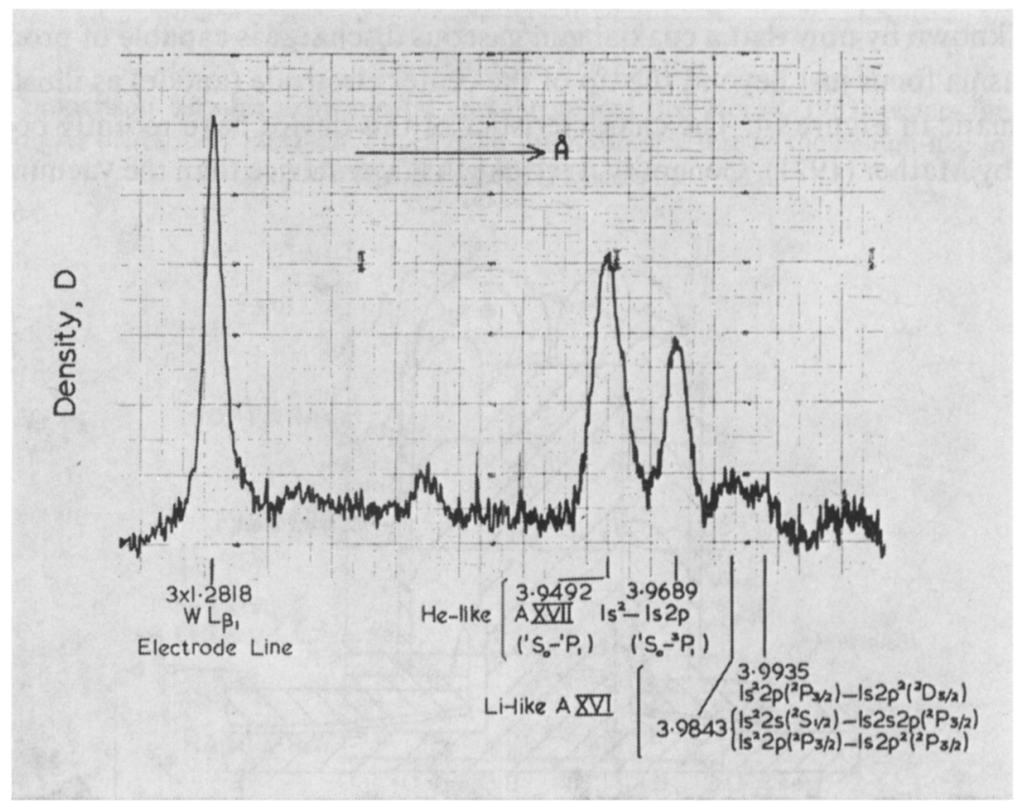

Fig. 15. Microdensitometer scan of spectrum obtained from coaxial gun plasma focus deuterium discharge seeded with $4 \%$ argon (from Peacock et al., 1971, Figure 8).

like ArXVII, as well as the lithium-like innershell transition line which again is the strongest of the innershell lines. This is as reported above for the vacuum spark, yet somewhat weaker for the lower- $Z$ argon which they explain as being due to a lower di-electronic recombination rate into A XVII. Attempts (Connerade et al., 1970) to introduce iron into the coaxial gun focus discharge have not resulted in ion stages above $\mathrm{xx}$.

(b) Continuum Radiation. As in the vacuum spark, $X$ rays with energy exceeding $500 \mathrm{keV}$ have been detected, and Lee et al. (1971 a) have succeeded in deducing an $\mathrm{X}$-ray continuum spectrum from electron tracks in nuclear emulsions, where the electrons are produced by photoelectric and Compton effects initiated by the incident $\mathrm{X}$-ray photons. Their spectrum is reproduced in Figure 16 along with a remarkably similar spectrum reported by Cline et al. (1968) for the solar flare hard X-ray impulses. 

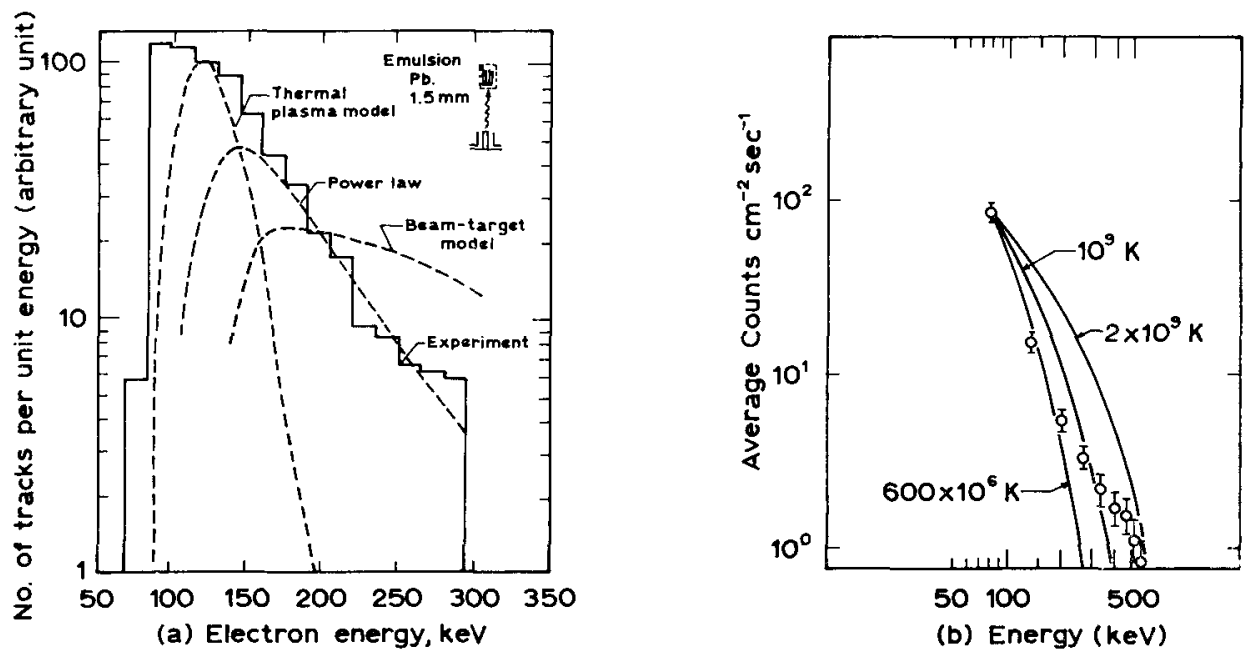

Fig. 16. Hard X-ray spectra obtained from: (a) coaxial-gun gaseous discharge focus (Lee et al., 1971a, Figure 2), and (b) solar flare (Cline et al., 1968, Figure 3).

\section{Summary}

With certain recent innovations, the vacuum spark has managed to keep pace with advances towards shorter and shorter wavelengths in the observation of solar flare phenomena. In spite of the large scaling factors involved due to the different length and time scales, the phenomenological similarities are striking as regards the spectra and time evolution. The coaxial gun plasma focus has also proven valuable as a gaseous discharge device and, while not producing the high stages of ionization in the heavy elements generated in the vacuum spark, is capable of producing a higher X-ray flux due to the increased size and energy.

\section{References}

Cline, T. L., Holt, S. S., and Hones, Jr., E. W.: 1968, J. Geophys. Res. Space Phys. 73, 434.

Cohen, L., Feldman, U., Swartz, M., and Underwood, J. H.: 1968, J. Opt. Soc. Am. 58, 843.

Connerade, J. P., Peacock, N. J., and Speer, R. J.: 1970, Solar Phys. 14, 159.

Doschek, G. A., Meekins, J. F., Kreplin, R. W., Chubb, T. A., and Friedman, H.: 1971, Astrophys. J. 170, 1573 .

Edlén, B.: 1947, Physica 13, 545; and other papers referenced here.

Elton, R. C.: 1968, Determination of Electron Temperatures between $50 \mathrm{eV}$ and $100 \mathrm{keV}$ from X-Ray Continuum Radiation in Plasmas, NRL Report 6738.

Flemberg, H.: 1942, Arkiv Mat. Astron. Fysik 28, 1.

House, L. L.: 1969, Astrophys. J. Suppl. 18, 21 ; also R. D. Cowan (private communication) for Tixix.

Kane, S. R. and Anderson, K. A.: 1970, Astrophys. J. 162, 1003; See also Kane, S. R., this issue, p. 822 .

Lee, J. H., Loebbaka, D. S., and Roos, C. E.: 1971a, Plasma Phys. 13, 347.

Lee, J. H., Shomo, L. P., Williams, M. D., and Hermansdörfer, H.: 1971b, Phys. Fluids 14, 2217.

Lie, T. N. and Elton, R. C.: 1971, Phys. Rev. A3, 865.

Lie, T. N. and Elton, R. C.: 1972, to be published.

Mather, J. W.: 1971, in R. H. Lovberg and H. R. Griem (eds.), Methods of Experimental Physics, -Plasma Physics, Vol. 9B, Chapt. 15, Academic Press, New York. 
Neupert, W. M. and Swartz, M.: 1970, Astrophys. J. Letters 160, L189.

Peacock, N. J., Speer, R. J., and Hobby, M. G.: 1969, J. Phys. B. 2, 798.

Peacock, N. J., Hobby, M. G., and Morgan, P. D.: 1971, Proceedings Fourth Conference on Plasma Physics and Controlled Nuclear Fusion Research, Madison, Wisconsin. IAEA, Vienna 1, 537.

\section{DISCUSSION}

S. $R$. Kane: Do you observe any radio (microwave) emission at the time of the hard X-ray emission from the laboratory plasma?

R. C. Elton: Yes, emission is observed predominantly at X-band wavelengths (frequency $\approx 10 \mathrm{GHz}$ ) and rises considerably with the X-ray burst.

C. de Jager: What is the mechanism of the observed microwave emission? Are magnetic fields involved?

R. C. Elton: The mechanism is not yet understood and the measurements are very preliminary. The magnetic fields near the compressed plasma are estimated (from pressure balance) to be $10^{7} \mathrm{G}$. It does not appear, so far, that the microwave wavelength varies with reduced discharge current.

B. S. Fraenkel: Is there some correlation between maximum current and spectroscopic results. Is something known about variation of the size of the instability?

R. C. Elton: We have not made a detailed study of the current dependence of the spectroscopic measurements. There is some evidence that the size of the concentrated plasma region tends to increase with peak current. 